\title{
ALTERACIÓN POSTURAL EN SEDESTACIÓN Y SU ASOCIACIÓN AL DOLOR LUMBAR EN EL PERSONAL ADMINISTRATIVO DEL HOSPITAL III DANIEL ALCIDES CARRIÓN - ESSALUD TACNA 2017
}

\author{
POSTURAL ALTERATION IN SEDESTATION AND ITS ASSOCIATION TO LUMBAR PAIN IN THE \\ ADMINISTRATIVE STAFF OF HOSPITAL III DANIEL ALCIDES CARRIÓN - ESSALUD TACNA 2017
}

\author{
Maribel Quispe Parra ${ }^{1}$ \\ Víctor A. Arias Santana ${ }^{2}$ \\ Presentado: 29/11/2018 \\ Aceptado: 27/05/2019 \\ Publicado online:17/07/2019
}

\section{RESUMEN}

Estudio realizado para medir si la alteración postural está asociado al dolor lumbar en el personal administrativo de los Hospital III Daniel Alcides Carrión Essalud Calana - Tacna 2017. Investigación observacional, analítica, prospectiva y de corte transversal, a nivel relacional. Se trabajó con el total del personal administrativo del Hospital III Daniel Alcides Carrión Essalud Calana - Tacna 2017, que cumplieron con los criterios de inclusión y exclusión de quienes se recopiló los datos de la ficha postural de sedestación, validada debidamente y llenada por los participantes. Se halló que en las primeras 10 horas de la mañana, la postura intermedia y la anterior es la que predomina en el aumento de dolor y en las siguientes horas es la postura anterior la que exacerba su dolor seguidamente de la intermedia. No existe diferencia significativa con la variable estado nutricional de los trabajadores y vinculación con el dolor lumbar. Hay una relación directamente proporcional entre intensidad del dolor y horas laborando ( $p: 0.03$ ). Se observa una diferencia significativa en la evolución del dolor a las 12:30 pm. Según sexo el dolor es mayor en el personal femenino, tiempo de servicio ( $p: 0.002$ ) y jornadas laborales tempranas especialmente en el personal con tiempo de servicio de 21 años a más.

Palabra Clave: Postura anterior, postura posterior y intermedia, dolor lumbar.

\footnotetext{
${ }^{1}$ Magíster en Investigación Científica e Innovación

2 Doctor en Ciencias y Magíster en salud pública
} 


\begin{abstract}
A study carried out to measure whether the postural alteration is associated with low back pain in the administrative staff of Hospital III Daniel Alcides Carrión Essalud Calana - Tacna 2017. Observational, analytical, prospective and cross-sectional research at the relational level. We worked with the total of the administrative staff of Hospital III Daniel Alcides Carrión Essalud Calana - Tacna 2017, who met the inclusion and exclusion criteria of those who collected the data of the postural tab for sitting, duly validated and filled out by the participants. It was found that in the first 10 hours of the morning, the intermediate and anterior positions predominate in the increase of pain and in the following hours it is the anterior position that exacerbates the pain followed by the intermediate one. There is no significant difference with the variable nutritional status of workers and linkage with low back pain. There is a directly proportional relationship between pain intensity and working hours ( $p: 0.03$ ). A significant difference is observed in the evolution of pain at 12:30 pm. According to sex, the pain is greater in the female staff, time of service ( $p: 0.002$ ) and early work days especially in the staff with service time of 21 years to more.
\end{abstract}

Keyword: Anterior position, posterior and intermediate posture, lumbar pain.

\title{
INTRODUCCIÓN
}

En la actualidad cada día se adoptan nuevos estilos de vida de acuerdo a la sociedad en la que cada uno se encuentra inmerso, de tal manera que pueda mejorar su calidad de vida. A pesar de ello, se ha dejado de lado una adecuada educación en higiene postural, hasta en cierto punto es menospreciado por el mismo desconocimiento de la importancia de esta. Una de las enfermedades laborales más comunes, es la lumbalgia, una de las causas de este dolor es el mucho tiempo en posturas inadecuadas. "El estrés laboral es el factor más común que pone a las personas en riesgo de sufrir dolores lumbares" (1). La postura correcta desde el punto de vista fisiológico es aquella que no es fatigante, no es dolorosa, no altera el equilibrio, el ritmo, ni la movilidad humana. Distinguimos 3 posturas: Bipedestación, Sedestación: anterior, intermedia y posterior, Decúbito: supino, prono y lateral (2). En la posición de sedestación el gasto energético, la demanda circulatoria y el trabajo muscular estático va a ser menor que en bipedestación. En esta postura el peso del tronco se traslada a la tuberosidad isquiática y por medio de ésta a los pies. La posición estática, en sedestación, de forma prolongada, junto con el movimiento repetido, propio de la utilización del teclado y el ratón; pone de relieve dos de los factores más importantes de riesgo músculo esquelético: el estatismo y el movimiento repetido. El dolor de origen lumbar se considera un problema de salud ocupacional, según Ceprit (Essalud) (3), ocupando el 2do. lugar en enfermedades ocupacionales (19.2\%). Aníbal Hermoza(4), sostuvo que al menos un millón de trabajadores en el Perú sufren afección en la zona lumbar de la columna vertebral lo que provoca que el 35\% de inasistencias en las áreas administrativas. En la práctica laboral se ha observado que los trabajadores administrativos que laboran en el Hospital III Daniel Alcides Carrión Essalud, Calana, tienen turnos de ocho horas con posturas alteradas en sedestación que son modificadas constantemente por la larga jornada laboral. Factores presentes como el sobrepeso, el sedentarismo y los asientos inadecuados carentes de una ergonomía adecuada son características a ser estudiadas.

Makhsous $\mathrm{M}$ et al (5) refiere que el estar sentado puede inducir la rotación posterior de la pelvis, la reducción de la lordosis lumbar, y el aumento de la tensión muscular, la presión del disco, y la presión sobre el isquion y el cóccix, que puede estar asociado con dolor de espalda baja. Lengsfeld et al. (6). afirma que un concepto de inclinación sincronizada con una inclinación posterior del asiento mientras 
que el respaldo está reclinado es preferible desde el punto de vista de la cinemática de la columna lumbar. Zemp (7) refiere que cada vez más personas pasan la mayor parte de su vida laboral sentados en una silla de oficina. Asimismo, afirma que existe una amplia gama de adaptación de asientos a través de la modificación de la postura silla y opciones de asientos dinámicos que proporcionan una función clave en la reducción o incluso prevenir el dolor de espalda causado por la prolongada sesión estática. Lis AM et al (8) concluye que el dolor lumbar (lumbalgia) ha sido identificado como uno de los trastornos más costosas entre la población trabajadora en todo el mundo. Refiere que estar sentado por más de la mitad de un día de trabajo, en combinación con posturas forzadas, hace aumentar la probabilidad de tener dolor lumbar y / o ciática. Santiago Bazán (9) refiere que el manejo debe ser multidisciplinari y que las posturas de trabajo mantenidas y la carga de peso son predisponentes para el desarrollo del dolor lumbar. Casas Sánchez et al (10) aconseja que adicionalmente, los factores psicológicos deben ser considerados para comprender el problema del dolor en cuello y espalda. Sánchez, E. (11)) encontró que el sexo femenino 80 \% es el más propenso al presentar dolor lumbar. Sanabria León afirmo que el dolor lumbar tiene un alto impacto en la calidad de vida del personal. La exposición a factores de riesgo biomecánico se relaciona entre otros con la estimación del dolor lumbar afectando su calidad de vida. (12).

Las posturas perpetuadas en el tiempo a través de la repetición o del mantenimiento sostenido nos lleva a una organización específica de la estática del cuerpo, que generará desalineaciones a nivel articular que en forma individual o en su sumatoria darán el resultado de las Alteraciones Posturales. (13). EN posición sentado, el centro de gravedad se sitúa detrás de las tuberosidades isquiáticas. Hay un aumento de la lordosis lumbar o retroversión de la pelvis (14). Las partes blandas como los músculos no nos dan el apoyo necesario y el único que podría cumplir esa función es el hueso más próximo a estos tejidos, resultando que en esa posición se comprimirían más lo tejidos blandos, como músculos y los nervios (15) Lo correcto es buscar que la columna vertebral debe posicionarse dentro de una mínima flexión y mínima extensión no llegando a los rangos máximo de flexión y extensión teniéndose en cuenta que mantener posturas mantenidas por mucho tiempo en el rango máximo generara alteraciones por la falta de control de movimiento y stress por el esfuerzo elevado de los músculos que mantiene la postura (16) Al estar en posición de sedestación por la biomecánica entendemos que el dolor que se produce no se debe solo al cansancio de los músculos (17) Las cargas inadecuadas están asociadas a la deformación mecánica (18). El síndrome postural es la deformación mecánica (nocicepción mecánica) de origen postural de los tejidos blandos normales y sanos puestos en tensión por malas posturas mantenidas (19).

\section{OBJETIVOS}

a) Identificar la alteración postural, en sedestación, (anterior, intermedia y posterior) que más produce el dolor lumbar según la hora de jornada laboral en el personal administrativo de Essalud - Tacna 2017.

b) Clasificar la intensidad del dolor lumbar en la escala de EVA (escala visual analógica del dolor) según la hora de jornada laboral en el trabajo.

c) Evaluar el nivel de asociación de la alteración postural en sedestación con el nivel de intensidad de dolor lumbar, según las condiciones personales y laborales.

\section{METOdOLOGÍA}

Estudio observacional, prospectivo de corte transversal y nivel relacional. Se realizó en el Hospital III Daniel Alcides Carrión del Distrito de Calana de la Ciudad de Tacna en el 2017. La población de estudio fueron 58 trabajadores administrativos. Se utilizó la escala visual analógica (EVA) para medir la intensidad del dolor. Se utilizó también una ficha de evaluación postural en sedestación registrada cada media hora según la postura y la escala del dolor referida. 


\section{RESULTADOS}

En la Tabla 1 podemos observar que en las primeras horas de la mañana la postura intermedia es la que predomina conforme pasan las horas, la postura anterior es la que el trabajador está adoptando hasta aproximadamente a partir de las 10 horas y en las siguientes horas laborales el predominio es la postura anterior seguida de la intermedia hasta el final de la jornada laboral.

La evolución de la intensidad del dolor lumbar es progresiva conforme avanza la jornada laboral. Existe una asociación entre horas de labor ejecutada e intensidad del dolor. A más horas de labor la intensidad aumenta ( $p$ : 0.03). Podemos afirmar con un intervalo de confianza del 95\% que existe una relación directa entre dolor y horas de labor. (Fig. 1)

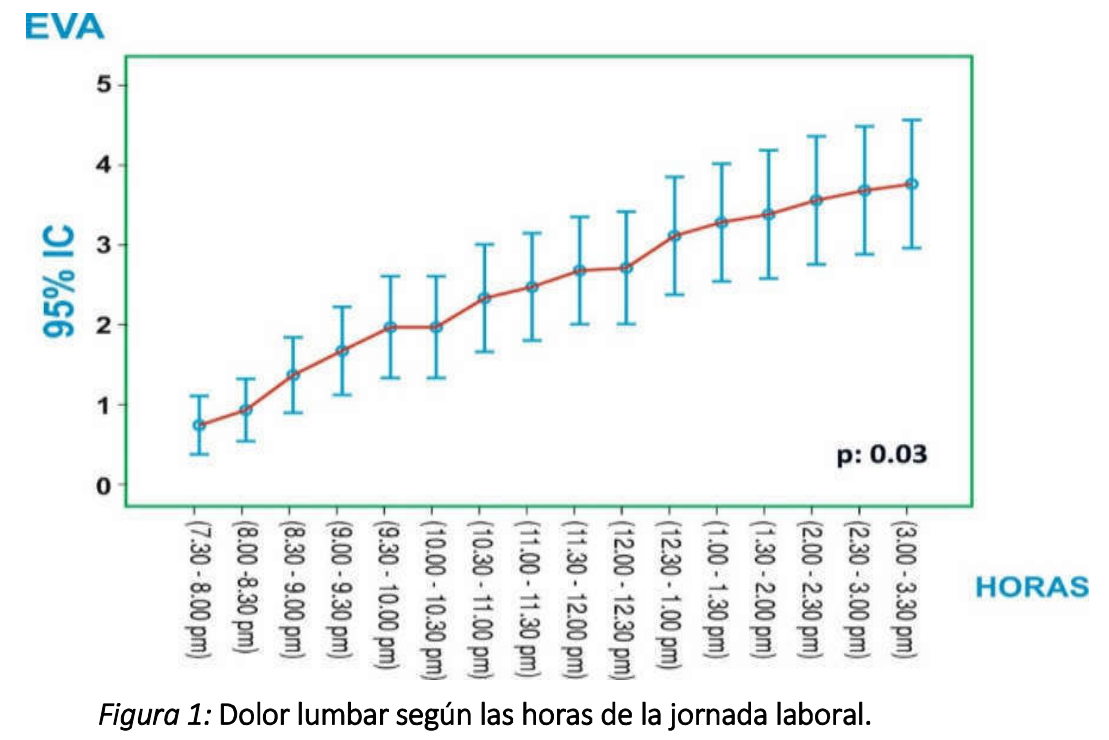

Al iniciar la jornada la postura intermedia es la que manifiesta menor tendencia de evolución del dolor con escalas bajas en cambio la postura anterior y la posterior zona más asociada a niveles de dolor que van evolucionando escalas mayores según las horas de jornada laboral. La evolución del dolor entre las 12.00 y $12: 30 \mathrm{pm}$, la postura más asociada a dolor va a ser la anterior seguida de la intermedia. El nivel más bajo de producción del dolor estuvo presente en el personal con posturas posteriores. Al finalizar la jornada el nivel de dolor más alto estuvo asociado a la postura anterior seguida de la postura intermedia. Los niveles más bajos de dolor se concentraron en la postura posterior.

No existe diferencia significativa entre los diferentes estados nutricionales de los trabajadores y vinculación con el dolor. Todos presentan la misma probabilidad de sufrir el daño.

Según sexo podemos afirmar que no existe diferencia significativa de la evolución del dolor lumbar hasta antes de las 12:30 pm. Esta diferencia se hace significativa a partir de las 12:30 pm donde la evidencia del dolor es mucho mayor en el personal femenino mostrando niveles de dolor en la escala de 4 a 5 . ( $p<0.05)$. Existe una diferencia significativa de la intensidad del dolor lumbar, según tiempo de servicio (p: 0.02). Se puede observar que se hace más evidente la manifestación de dolor lumbar en las jornadas tempranas laborales, en el personal con un tiempo de servicio de 21 años a más.

\section{DISCUSIÓN}

Se pudo observar que, en sedestación, en una jornada de 8 horas de 7:30 am. a 3:30 pm., es en las primeras horas, a partir de las 10 de la mañana la postura anterior y la posterior quienes predominan en el aumento del dolor y en las siguientes horas es la postura anterior la que exacerba su dolor 
seguidamente la postura intermedia. Lengsfeld et al (6) en el 2000 sostiene que una sentada prolongada es un factor de riesgo para el factor lumbar y sugiere que se debe mantener una postura intermedia durante la sedestación. Zemp et al (7) en el 2013 menciona que el dolor lumbar está asociada a una postura estática pero que cambiando regularmente la posición sentada ayuda a reducir el dolor. Lis A.M. et al (8) en el 2007 concluye que no solo la sedestación se asocia al dolor lumbar sino estar sentado por más de la mitad de un día de trabajo esto hace aumentar la probabilidad del dolor lumbar. Encontramos que no existe diferencia significativa con la variable estado nutricional de los trabajadores y vinculación con el dolor; si bien el índice de masa corporal es un indicador en la evaluación del dolor lumbar desde el punto de vista significativo no está relacionado, lo que también sostiene Bazán S. et al (9). en el año 2012 se observa una diferencia significativa de la evolución del dolor, especialmente a partir de las 12:30 pm. Según sexo, se evidencia que el dolor es mayor en el personal femenino. Casas $\mathrm{S}$ et al (10) en el 2004 encontró también una asociación de dolor lumbar entre el sexo femenino en horas de la semana en posición sedente asociado a la falta de actividad física. Sánchez et al (11) en el 2015 concluye en su estudio que el sexo femenino es el más propenso al presentar dolor lumbar.

En el grupo de estudio existe una diferencia significativa del dolor según tiempo de servicio ( $p$ : 0.002), observamos más evidencia de dolor en las jornadas tempranas laborales en el personal con tiempo de servicio de 21 años a más, no existiendo esto en los demás grupos. Sanabria AM et al (12) en el año 2014-2015 estableció la percepción del personal de enfermería según el tipo de contrato a término indefinido, favoreciendo la ausencia del dolor lumbar, pero tomándose en cuenta la consideración de las exigencias físicas como altas y muy altas por parte del trabajador lo cual debe ser asociado con mayor prevalencia de dolor de acuerdo a las características propias como la edad y el tiempo de servicio del trabajador. Esto nos lleva a la reflexión que los resultados obtenidos dan cuenta que existe un problema de salud considerable en los trabajadores de la empresa generando posturas de riesgo a raíz de su propio desempeño laboral, haciéndolo más vulnerable a padecer algún tipo de discapacidad en el futuro.

Se recomienda realizar trabajos de investigación de la misma característica en personal asistencial, diferenciando por servicio de atención. Proponer un Plan de prevención y tratamiento en la institución con enfoque en personal administrativo al comienzo y posteriormente en personal asistencial y proponer en base a los resultados de esta investigación a las autoridades de Essalud capacitaciones de posturas ergonómicas y pautas activas dirigidas por el área de salud ocupacional con la participación de un fisioterapeuta, para poder prevenir a tiempo los casos de dolor lumbar y otros que se presenten.

\section{REFERENCIAS BIBLIOGRÁFICAS}

1. Martínez Pérez Viviana Lumbalgia, una enfermedad laboral común, @El UniversalCtg. 10 de junio de 2011. http://www.eluniversal.com.co/salud/lumbalgia-una-enfermedad-laboral-comun28555-KREU107368.

2. Laborales pdr. labor salus.com. [Online].; 2013 [cited 2015. Available from:http://laborsalus.com/Documentacion/cmp_higienen_postural.pdf.

3. Yupanqui Godo L, Inoñan P, Gallegos L, Caro E, Chamochumbi W. Instituto Salud Y Trabajo. [Online].; $2011 \quad$ [cited $2015 . \quad$ Available from:http://www.usmp.edu.pe/recursoshumanos/pdf/1)\%20Diagnostico\%20de\%20Seguridad\%2 Oy\%20Salud\%20en\%20el\%20Peru.pdf.

4. Hermoza A. Diario La República. [Online].; 2010 [cited 2015. Available from: http://larepublica.pe/10-11-2010/cerca-de-un-millon-de-trabajadores-en-peru-sufre-lumbalgia.

5. Makhsous M, Lin F, Hendrix R, Hepler M, Zhang L. Sitting with adjustable ischial and back supports: biomechanical changes. PUBMED. 2003 junio; 28(11).

6. Lengsfeld M, Frank A, Van Deursen D, Griss P. Lumbar spine curvature during office chair sitting. Med. Ens Phys. 2000 noviembre; 22(9). 
7. Baumgartner D, Zemp R, List R, Stoop M, Naxera J. The spinal curvature of three different sitting positions analysed in an open MRI scanner. Scientif world Journal. 2012 noviembre; 25.

8. Lis M, Black K, Korn H, Nordin M. Association between sitting and occupational LBP. Eur Spine. 2007 feb; 16(2).

9. Bazán Cristhian Santiago. Dolor miofascial lumbar en estudiantes de Terapia Física Rehabilitación de la Universidad Nacional Federico Villarreal. [Tesis de licenciatura 2012]. Perú. Universidad Nacional Federico Villarreal.

10. Casas Sánchez Aminta Stella, María Solange Patiño Segura, MS. Prevalencia y factores asociados con el dolor de espalda y cuello en estudiantes universitarios. Revista de la Universidad Industrial de Santander. Salud [Internet]. 2012; 44(2):45-55. Disponible en: http://www.redalyc.org/html/3438/343835705007/.

11. Sánchez Caja Evelyn. Concordancia inter-evaluadores en la palpación de puntos gatillo miofasciales en pacientes con lumbalgia realizado por estudiantes del último año de la carrera de terapia física. [Tesis de licenciatura]. Quito. Pontificia universidad católica del Ecuador 2015.

12. Sanabria León Alba María. Prevalencia del Dolor Lumbar y su relación con factores de riesgo biomecánico en persona de enfermería 2014-2015. Medicina (Bogotá) Vol. 37 No. 4 (111) Págs. 319-330 diciembre 2015.

13. Fernandez Gomez M, Gregori Torada E, Mondelo P. Ergonomía 4 el trabajo en oficinas. España: Barcelona; 2002.

14. Racero GA. I Congreso Iberoamericano de Fisioterapia y Kinesiología. [Online]. [cited 2015. Available from: http://www.sinergia-web.com/archivos/prev-correc_alt_posturales.pdf.

15. Ergonomía: el asiento parte 4. [Online]. [cited 2015. Available from: http://www.estrucplan.com.ar/Producciones/entrega.asp?ldEntrega=2399.

16. Mark Comerford, Sarah Mottram, Kinetic Control The management of Uncotrolled Movement. Edit. Elservier Australia 2012 pag,46-47.

17. Liebenson C. In Manual de Rehabilitación de la Columna Vertebral.: Paidratribo; 2008. p. 36-38.

18. Corre y Col FL. Manipulaciones Vertebrales. 2nd ed. Francia: Masson S.A; 1995.

19. Wits B. Método Mc Kenzie: curso A. 